\title{
Electrocatalysis on shape-controlled metal nanoparticles: progress in surface cleaning methodologies
}

\author{
M.A. Montiel, F. J. Vidal-Iglesias, V. Montiel, J. Solla-Gullón* \\ Instituto de Electroquímica, Universidad de Alicante, Apdo. 99, E-03080 Alicante, \\ Spain \\ *Corresponding author: jose.solla@ua.es
}

\begin{abstract}
The use of shape-controlled metal nanoparticles has produced not only a clear enhancement in the electrocatalytic activity of different reactions of interest but also a better understanding of the effect of the surface structure on nanoscaled materials. However, it is well-accepted that a correct understanding of the correlations between shape/surface structure and electrochemical reactivity indispensably requires the use of clean surfaces. In this regard, and considering that most of the synthetic methodologies available in the literature for the preparation of these shaped metal nanoparticles employ capping agents, the development of effective surface cleaning methodologies able to remove such capping agents from the surface of the corresponding nanoparticles, becomes an extremely important prerequisite to subsequently evaluate their electrocatalytic properties for any reaction of interest. Consequently, in this contribution, we summarize the most relevant advances about surface cleaning procedures applied to different shaped metal nanoparticles for electrocatalytic purposes. It is worth mentioning that this work will only include contributions in which the surface cleanness of the samples is specifically evaluated using well-established electrochemical tools.
\end{abstract}

Keywords: metal nanoparticles; shape-controlled; surface cleaning; Electrocatalysis.

\section{Introduction}

Since the first examples of the use of shape-controlled metal nanoparticles in Electrocatalysis, dealing with the application of cubic platinum (Pt) nanoparticles towards ammonia electrooxidation in alkaline solution [1*, 2], many contributions have been reported in this topic. Currently, the most relevant advances in this field appear now included in interesting reviews about the use of these shaped metal nanoparticles for many different electrocatalytic applications [3-12]. Interestingly, all these studies 
clearly point out that the surface structure, that is, the particular arrangement of the atoms at the surface of the shaped nanoparticles is the key parameter determining their resulting electrocatalytic properties/reactivity as previously described with metal single crystal electrodes [13, 14]. In this sense, the availability of shape-controlled metal nanoparticles is of outstanding importance because the shape of the nanoparticles essentially determines their surface structure, that is, their surface atomic arrangement and coordination. In fact, the shape of a nanoparticle anticipates its preferential surface structure due to the intrinsic correlation between the shape and the surface structure of a nanoparticle $\left[15,1^{\circ}\right]$. However, it is also worth noting that a perfect shape-surface structure correlation is unrealistic and consequently, even with a well-defined (in terms of size and shape) nanoparticle, its surface will be extremely complex containing not only some ordered surface domains of different dimensions but also a determined number of defect, corner, edge, step and kink sites, all of them contributing to the resulting electrocatalytic activity.

Many options are now available in the literature for the preparation of shape-controlled metal nanoparticles [5, 17-20], most of them based on the chemical reduction of a metallic precursor in the presence of a capping agent. Although other experimental parameters such as electrolyte, temperature, reducing time, nature of the reducing agent and others may also affect, the role of the capping agent is extremely important because it modifies the surface energies of the nanoparticles during their nucleation and growth steps thus overcoming the thermodynamic limitations and allowing metal nanoparticles with a particular shape to be obtained. The presence of these capping agents at the surface of the nanoparticles, however, precludes its direct application in Electrocatalysis, where it is well-established that having a clean surface is a requirement for a correct understanding of surface structure-electrocatalytic reactivity relationships. Consequently, once shaped metal nanoparticles are prepared, these must be inevitably submitted to specific decontamination protocols to remove the presence, even in residual amounts, of the capping agents at the surface of the nanoparticles. In addition, this surface cleaning must be performed without altering the initial surface structure of the nanoparticles. This is, from our point of view, one of the most critical aspects that have to be fully satisfied for the establishment of correct correlations between particle shape/surface structure and electrochemical reactivity with these systems. With this objective in mind, this contribution describes some specific decontamination protocols reported in literature and successfully applied for shape-controlled metal nanoparticles for subsequent electrocatalytic purposes. At this respect, it is important to note that a "universal" cleaning method is not possible and the particular cleaning will basically depend on two main parameters, a) the chemical 
nature of the capping agent and b) its interaction with the surface of the shaped nanoparticles this latter being determined by the nature of metal as well as by the structure of surface exposed. Figure 1 schematically illustrates the topic covered by the present work.

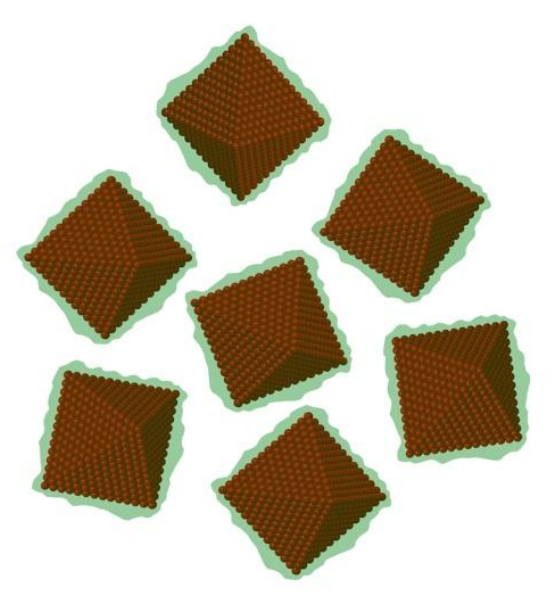

As-prepared shaped metal nanoparticles

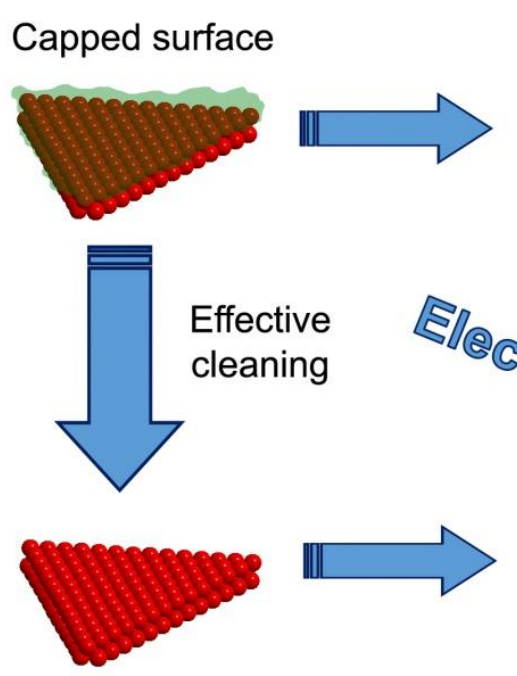

Clean surface
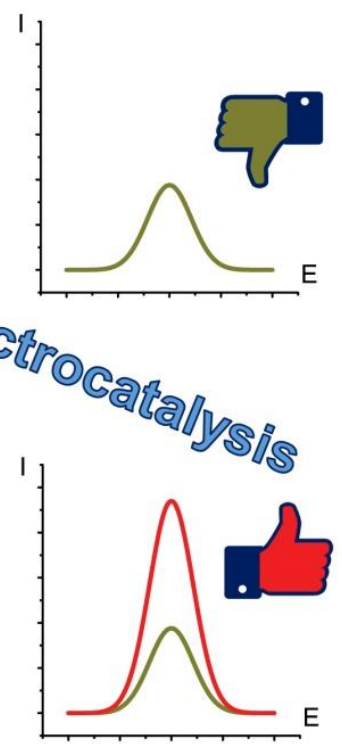

Figure 1. Schematically representation of the topic covered in this contribution.

Finally, it is also important to mention that some methodologies able to prepare shapecontrolled metal nanoparticles in the absence of capping agents are also available in the literature, including electrochemical methods [21], cathodic corrosion [22], surfactant-free solvothermal synthesis [23] or solid-state chemistry methods [24].

\section{Electrochemical reactions for evaluating the surface cleanness}

Taking in mind that the use of clean surfaces is a fundamental requisite to properly study the electrochemical properties of shaped metal nanoparticles, the question of how to evaluate this surface cleanness obviously emerges. This question can be again satisfied by exploring some of the interesting options that Surface Electrochemistry of metal single crystals provides us. For example, the so-called hydrogen region for $\mathrm{Pt}$ and palladium $(\mathrm{Pd})$ surfaces can be employed to properly evaluate the level of cleanness of the surface. In brief, if the different hydrogen and/or anion adsorptiondesorption states are well-defined (sharpness) and reversible (symmetry), there is a clear evidence of the adequate cleanness of the surface. For gold ( $\mathrm{Au}$ ) surfaces, the analysis of the oxide formation/reduction region can be also used to extract information about the surface cleanness. On the other hand, by using different underpotential deposition (UPD) processes, some information about the surface cleanness can be 
also extracted. Interestingly, a more detailed analysis of these processes can also provide other relevant electrochemical parameters such as surface area as well as qualitative and quantitative information of the different surface sites present at the surface of the nanoparticles. In fact, these electrochemical tools have been shown to be extremely sensitive to analyse possible perturbations of the surface structure of the nanoparticles when too aggressive decontamination protocols are employed. In this way, it was possible to evidence that the use of UV/ozone as decontamination protocol [25-27] resulted in an important surface structure perturbation but without affecting neither the size nor the shape of the nanoparticles as deduced from TEM measurements [28“0].

For shape-controlled metal alloy nanoparticles, indisputably one of the hot topics in Electrocatalysis $\left[4,9,11,12,2^{\circ}, 30\right]$, the evaluation of the surface cleanness becomes much more complex due to the intrinsic complexity of the alloy surfaces [31-33]. In these systems, one should verify, using some of the previously mentioned electrochemical tools, the correct surface cleanness for the corresponding controlling metal, for instance Pt for Pt-based alloys, obviously prepared using similar experimental conditions and then simply assuming the effectiveness of the applied cleaning procedure. Without this verification, the surface cleanness of the shapecontrolled metal alloy nanoparticles cannot be warranted.

\section{Surface cleaning methodologies}

In the following, we will briefly describe the most relevant decontamination protocols found in the literature and applied to different shaped metal nanoparticles for various electrocatalytic purposes.

Cubic, cuboctahedral and tetrahedral-octahedral Pt nanoparticles prepared in the presence of sodium polyacrylate (NaPA) were decontaminated by applying a $\mathrm{NaOH}$ cleaning [34*0]. The surface cleaning was achieved by the addition of some $\mathrm{NaOH}$ pellets to the colloidal suspension which induce the precipitation of the nanoparticles. After collecting and washing the nanoparticles with ultrapure water, the sample was ready for electrochemical measurements. The effectiveness of the cleaning was evidenced by the definition and reversibility of the so-called hydrogen features regardless of the used supporting electrolyte $\left(\mathrm{H}_{2} \mathrm{SO}_{4}, \mathrm{HClO}_{4}\right.$ or $\left.\mathrm{NaOH}\right)$. Interestingly, this simple decontamination was shown to be also effective with cubic Pd nanoparticles prepared in the presence of cetyltrimethylammonium bromide (CTAB) [35, 36] and cubic $\mathrm{Pt}$ nanoparticles prepared in the presence of tetradecyltrimethylammonium bromide (TTAB) [37, 38]. However, this cleaning resulted ineffective with shaped Pt 
nanoparticles prepared with polyvinylpyrrolidone (PVP) [37, 39] which is one of the most usual capping agents for the preparation of shape-controlled metal nanoparticles [40]. To the best of our knowledge, the first example of clean shape-controlled $\mathrm{Pt}$ nanoparticles prepared with PVP was reported by Monzó et al. [41*0]. They proposed the use of $\mathrm{H} \mathrm{H}_{2} \mathrm{O}_{2} / \mathrm{H}_{2} \mathrm{SO}_{4}$ solution to remove the PVP. They stated that the oxygen bubbling coming from the $\mathrm{H}_{2} \mathrm{O}_{2}$ decomposition was the cleaning agent, thus taking place a physical removal process of the PVP. With a similar concept, Levendorf et al. [42] obtained clean cubic and octahedral/tetrahedral Pt nanoparticles prepared with PVP using an adapted liquid phase UV photo-oxidation (UVPO) technique. In these two last cases, the voltammetric responses of the shaped $\mathrm{Pt}$ nanoparticles in $0.5 \mathrm{M}$ $\mathrm{H}_{2} \mathrm{SO}_{4}$ were very similar to those reported with "clean" cubic and octahedral/tetrahedral Pt nanoparticles thus pointing out the effectiveness of their methodologies.

Interestingly, Yang et al. [43] proposed a different procedure to remove commonly used capping agents, such as PVP and oleylamine/oleic acid, during the synthesis of shapecontrolled $\mathrm{Pt}$ nanoparticles. This method consisted in the use of a simple electrochemical potential cycling in which the gold substrate on which the nanoparticles were deposited was cycled between 0 and $1.0 \mathrm{~V}$ (vs $\mathrm{RHE}$ ) in $0.5 \mathrm{M} \mathrm{NaOH}$ with a scan rate of $0.5 \mathrm{~V} \mathrm{~s}^{-1}$. After 100 cycles, the cleanness was evaluated by transferring the electrode to a second electrochemical cell containing $0.5 \mathrm{M} \mathrm{H}_{2} \mathrm{SO}_{4}$. From the obtained voltammetric response it was concluded that both PVP and oleylamine/oleic acid could be successfully removed from the surface of the nanoparticles with this electrochemical potential cycling methodology performed in $0.5 \mathrm{M} \mathrm{NaOH}$ solution. However, and despite the effectiveness of this "electrochemical cleaning procedure", this method has some practical limitations, particularly for large amounts of nanoparticles.

To overcome this limitation, Zalineeva et al. $[44,45]$ proposed an easy methodology to obtain clean shape-controlled Pd nanoparticles prepared in the presence of PVP by using an optimised $\mathrm{NaOH}$ treatment in contrast to previous unsuccessful contributions $[38,46]$. Briefly, the PVP-colloidal suspension was first diluted with $\mathrm{H}_{2} \mathrm{O}$ and the precipitation of the shaped $\mathrm{Pd}$ nanoparticles reached by the addition of $\mathrm{NaOH}$ pellets until having a $1 \mathrm{M} \mathrm{NaOH}$ solution (this $\mathrm{NaOH}$ concentration is significantly higher than that previously used). The nanoparticles were then collected by precipitation and their corresponding voltammetric profile in $0.5 \mathrm{M} \mathrm{H}_{2} \mathrm{SO}_{4}$ displayed well-defined voltammetric features thus denoting the effectiveness of the surface cleaning.

Neergat and co-workers reported two different alternatives for the removal of stabilizers and capping agents with shape-controlled $\mathrm{Pd}$ nanoparticles prepared in the presence of PVP and others additives such as $\mathrm{Br}^{-}, \mathrm{Cl}^{-}$and citrate ions $[47,48]$. In the first 
approach [47], cubic Pd nanoparticles were treated with tert-butylamine (TBA) during three days under continuous stirring after which the samples were collected by centrifugation and redispersed in ethanol. The sample was then electrochemically characterized in $0.1 \mathrm{M} \mathrm{HClO}_{4}$ and the results pointed out the effectiveness of the proposed cleaning methodology. Subsequently, in this case for various shapecontrolled $\mathrm{Pd}$ nanoparticles including cubic, truncated-octahedral and cubic/cubooctahedral nanoparticles, a $\mathrm{NaBH}_{4}$ solution was employed for the removal of the different additives [48]. Thus, after washing the samples with acetone and ethanol/hexane mixtures, the nanoparticles were dispersed in a water solution containing $\mathrm{NaBH}_{4}$ (60 mg of $\mathrm{NaBH}_{4}$ in $10 \mathrm{~mL}$ of water). The solution was then sealed, sonicated for $30 \mathrm{~min}$ and heated at $85^{\circ} \mathrm{C}$ under stirring for $8 \mathrm{~h}$. The nanoparticles were finally collected by centrifugation and electrochemically evaluated in $0.1 \mathrm{M} \mathrm{HClO}_{4}$. The reported cyclic voltammograms showed an improved surface cleanness although a full PVP removal was not achieved as deduced from $\mathrm{CHN}$ analyses, from which about a $90 \%$ cleaning is estimated. In both decontamination procedures, the improvement in surface cleanness produced clear enhanced ORR activities.

Luo et al. [49] applied a combined TBA/ $\mathrm{NaBH}_{4}$ cleaning to get clean Pt-Pd nanocubes prepared in the presence of PVP. The removal of the PVP was carried out by dispersing the nanoparticles in a TBA/NaBH 4 aqueous solution $\left(\mathrm{NaBH}_{4} / \mathrm{TBA} /\right.$ water, 1.9 $\mathrm{mg} / 20 \mathrm{~mL} / 5 \mathrm{~mL}$ ) for 30 min under continuous stirring and at room temperature. After that, the sample was collected by centrifugation and washed with an ethanol/acetone mixture to remove the excess of amines. Raman and FTIR analyses suggest the effective removal of the PVP from the surface of the Pt-Pd nanocubes with the combined TBA/NaBH${ }_{4}$ cleaning in comparison with the separately TBA and $\mathrm{NaBH}_{4}$ treated samples. As a result, the electrocatalytic activity of the clean $\mathrm{Pt}-\mathrm{Pd}$ nanocubes towards methanol oxidation in $0.1 \mathrm{M} \mathrm{HClO}_{4}$ showed an evident enhancement.

More recently, Arán-Ais et al. [50'] reported a new methodology to clean various shape-controlled Pt nanoparticles prepared using oleylamine/oleic acid as capping agent/solvent. The freshly prepared nanoparticles were initially washed with a hexane/ethanol mixture. Then, the sample was redispersed in $20 \mathrm{~mL}$ of methanol after which a pellet of $\mathrm{NaOH}$ was added to the solution and sonicated for $5 \mathrm{~min}$. After the nanoparticles precipitated, the alkaline solution of methanol was removed and the nanoparticles washed with acetone. This treatment was repeated at least three times, after which the sample was washed and stored with ultrapure water. The different shaped Pt nanoparticles were electrochemically characterized in much detail and, 
particularly, their voltammetric profiles in $0.5 \mathrm{M} \mathrm{H}_{2} \mathrm{SO}_{4}$ were clear evidences of the effective surface cleanness.

On the other hand, for shape-controlled Au nanoparticles, it has been stated that the electrochemical deposition of a film of $\mathrm{PbO}_{2}$ in alkaline solution $[51,52]$ is very effective in order to remove different capping agents, including polyethylene glycol dodecyl ether (Brij $\left.{ }^{\circledR} 30\right)$, CTAB and poly(diallyldimethylammonium chloride) (PDDA)). Interestingly, the $\mathrm{NaOH}$ cleaning was shown also effective to get clean cubic and octahedral $\mathrm{Au}$ nanoparticles prepared with CTAB $[53,54]$ which were used to electrochemically oxidise different organic molecules such as methanol, ethanol, formaldehyde and glycerol.

To end this contribution, Figure 2 collects some of the characteristic voltammetric profiles of clean shape-controlled $\mathrm{Pt}, \mathrm{Pd}$ and $\mathrm{Au}$ nanoparticles reported in the literature. Each voltammogram displays distinct features associated with their corresponding preferential surface structure. For a more detailed analysis, we refer to Refs 34,45 , and 55.

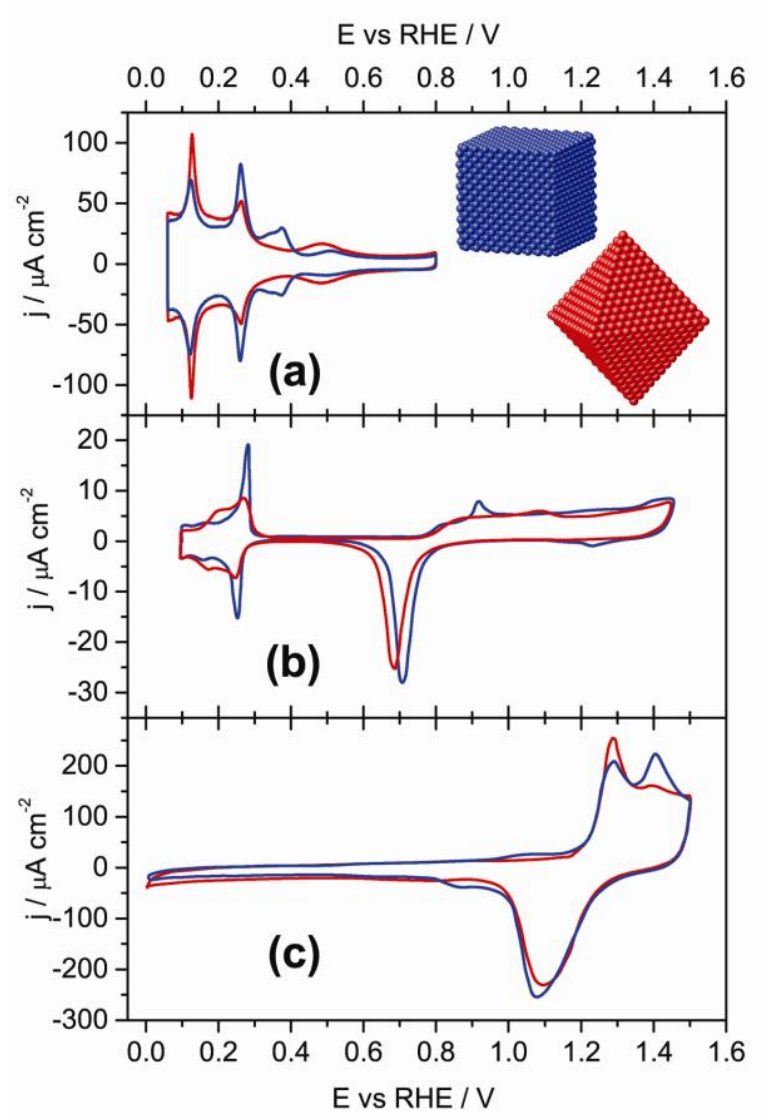

Figure 2. Representative voltammetric profiles of clean cubic (blue lines) and octahedral (red lines) $\mathrm{Pt}(\mathrm{a}), \mathrm{Pd}(\mathrm{b})$ and $\mathrm{Au}(\mathrm{c})$ nanoparticles obtained in $0.5 \mathrm{M} \mathrm{H}_{2} \mathrm{SO}_{4}(\mathrm{a}, \mathrm{b})$ and $0.1 \mathrm{M} \mathrm{NaOH}$ 
(c) at $50 \mathrm{mV} \mathrm{s}^{-1}(\mathrm{a}, \mathrm{c})$ and $5 \mathrm{mV} \mathrm{s}^{-1}$ (b). Data extracted from references 34 (figure a), 45 (figure b) and 54 (figure $\mathrm{c}$ ).

\section{Summary}

By using shape-controlled metal nanoparticles, a clear electrocatalytic enhancement on several electrochemical reactions of interest has been reported. However, and due to the fact that most of the routes available for the preparation of these shaped nanoparticles require capping agents, the development of effective surface cleaning methodologies is unavoidable before their use as electrocatalysts. In this contribution, we have summarized some of the optimal decontamination procedures existing in literature. In this way, shaped $\mathrm{Pt}, \mathrm{Pd}$ and $\mathrm{Au}$ nanoparticles prepared using some of most conventional capping agents such as NaPA, PVP, CTAB, TTAB and oleylamine/oleic acid can be properly cleaned and adequately used for different electrochemical reactions of interest.

\section{Acknowledgments}

This work has been financially supported by the MINECO of Spain through project CTQ2013-48280-C3-3-R.

\section{References}

Papers of particular interest, published within the period of review, have been highlighted as:

- paper of special interest

-• paper of outstanding interest

[1] F.J. Vidal-Iglesias, J. Solla-Gullón, P. Rodríguez, E. Herrero, V. Montiel, J.M. Feliu, A. Aldaz, Shape-dependent electrocatalysis: ammonia oxidation on platinum nanoparticles with preferential (100) surfaces, Electrochem. Commun. 6 (10) (2004) 1080-1084.

-. This paper reported the first case of the application of shape-controlled metal nanoparticles in Electrocatalysis. 
[2] J. Solla-Gullón, F.J. Vidal-Iglesias, P. Rodríguez, E. Herrero J.M. Feliu, J. Clavilier, A. Aldaz, In situ surface characterization of preferentially oriented platinum nanoparticles by using electrochemical structure sensitive adsorption reactions, J. Phys. Chem. B 108 (2004) 13573-13575.

[3] M.T.M. Koper, Structure sensitivity and nanoscale effects in electrocatalysis, Nanoscale 3 (2001) 2054-2073.

[4] J. Solla-Gullón, F.J. Vidal-Iglesias, J.M. Feliu, Shape dependent electrocatalysis. Annu. Rep. Prog. Chem., Sect C 107 (2011) 263-297.

[5] B. Wu, N. Zheng, Surface and interface control of noble metal nanocrystals for catalytic and electrocatalytic applications. Nano Today 8 (2013) 168-197.

[6] H. You, S. Yang, B. Ding, H. Yang, Synthesis of colloidal metal and metal alloy nanoparticles for electrochemical energy applications. Chem .Soc. Rev. 42 (2013) 2880-2904.

[7] S.E.F. Kleijn, S.C.S. Lai, M. T. M. Koper, P. R. Unwin, Electrochemistry of Nanoparticles, Angew. Chem. Int. Ed. 53 (2014) 3558-3586.

[8] H. Lee, Utilization of shape-controlled nanoparticles as catalysts with enhanced activity and selectivity, RSC Advances 4 (2014) 41017-41027.

[9] F.J. Vidal-Iglesias, J. Solla-Gullón, J.M. Feliu, Recent advances in the use of shapecontrolled metal nanoparticles in electrocatalysis, in: I.K. Ozoemena, S. Chen (Eds.), Nanomaterials for Fuel Cell Catalysis, Springer International Publishing, 2016, pp. 3192.

[10] S. Cao, F.F. Tao, Y. Tang, Y. Li, J. Yu, Size-and shape-dependent catalytic performances of oxidation and reduction reactions on nanocatalysts. Chem. Soc. Rev., 45 (2016) 474-4765.

[11] Y. Kang, P. Yang, N. M. Markovic, V. R. Stamenkovic, Shaping electrocatalysis through tailored nanomaterials, Nano Today 11 (2016) 587-600.

[12] J.W. Hong, Y. Kim, Y. Kwon, S. W. Han, Noble Metal Nanocrystals with Controlled Facets for Electrocatalysis, Chem. Asian J. 11 (2016) 2224-2239.

[13] M.T.M. Koper, Fuel Cell Catalysis: A Surface Science Approach. Electrocatalysis and Electrochemistry. John Wiley \& Sons, Hoboken, New Jersey, 2009.

[14] V. Climent, J.M. Feliu, Thirty years of platinum single crystal electrochemistry. J Solid State Electrochem 15 (2011)1297-1315. 
[15] N. Tian, Z.Y. Zhou, S.G. Sun, Platinum Metal Catalysts of High-Index Surfaces: From Single-Crystal Planes to Electrochemically Shape-Controlled Nanoparticles. J. Phys. Chem. C 112 (2008) 19801-19817.

[16] Z.Y. Zhou, N. Tian, Z.Z. Huang, D.J. Chen, S.G. Sun, Nanoparticle catalysts with high energy surfaces and enhanced activity synthesized by electrochemical method, Faraday Discuss. 140 (2009) 81-92.

- This paper visually correlates the particle shape (for fcc metal nanoparticles) with their surface structure.

[17] Y. Xia, Y. Xiong, B. Lim, S.E. Skrabalak, Shape-controlled synthesis of metal nanocrystals: Simple chemistry meets complex physics?, Angew. Chem. Int. Ed. 48 (2009) 60-103.

[18] G.J. Leong, M.C. Schulze, M.B. Strand, D. Maloney, S.L. Frisco, H.N. Dinh, B. Pivovar, R.M. Richards, Shape-directed platinum nanoparticle synthesis: Nanoscale design of novel catalysts, Appl. Organomet. Chem. 28 (2014) 1-17.

[19] Y. Xia, X. Xia, H.C. Peng, Shape-Controlled Synthesis of Colloidal Metal Nanocrystals: Thermodynamic versus Kinetic Products, J. Am. Chem. Soc., 137 (2015) 7947-7966

[20] K.D. Gilroy, A. Ruditskiy, H.C. Peng, D. Qin, Y. Xia, Bimetallic Nanocrystals: Syntheses, Properties, and Applications, Chem. Rev. 116 (2016) 10414-10472.

[21] N. Tian, Z. Y. Zhou, S.G. Sun, Y. Ding, Z.L. Wang, Synthesis of Tetrahexahedral Platinum Nanocrystals with High-Index Facets and High Electro-oxidation Activity Science 316 (2007) 732- 735.

[22] M. Duca, P. Rodriguez, A. I. Yanson, M.T.M. Koper, Selective Electrocatalysis on Platinum Nanoparticles with Preferential (100) Orientation Prepared by Cathodic Corrosion, Top. Catal., 57 (2014) 255-264.

[23] C. Gumeci, A. Marathe, R.L. Behrens, J. Chaudhuri, C. Korzeniewski, Solvothermal synthesis and electrochemical characterization of shape-controlled $\mathrm{Pt}$ nanocrystals. J Phys Chem C 118 (2014) 14433-14440.

[24] C. Zhang, S.Y. Hwang, A. Trout, Z. Peng, Solid-state chemistry-enabled scalable production of octahedral $\mathrm{Pt}-\mathrm{Ni}$ alloy electrocatalyst for oxygen reduction reaction. J Am Chem Soc. 136 (2014):7805-7808 
[25] C. Aliaga, J.Y. Park, Y. Yamada, H.S. Lee, C.K. Tsung, P. Yang, G.A. Somorjai Sum frequency generation and catalytic reaction studies of the removal of organic capping agents from Pt nanoparticles by UV-Ozone treatment. J. Phys. Chem. C 113 (2009) 6150-6155.

[26] J.M. Krier, W.D. Michalak, L.R. Baker, K. An, K. Komvopoulos, G.A. Somorjai, Sum frequency generation vibrational spectroscopy of colloidal platinum nanoparticle catalysts: Disordering versus removal of organic capping, J. Phys. Chem. C 116 (2012) 17540-17546.

[27] M. Crespo-Quesada, J.M. Andanson, A. Yaruli, B. Li, Y. Xia, L. Kiwi-Minsker, UVozone cleaning of supported poly(vinylpyrrolidone)-stabilized palladium nanocubes: Effect of stabilizer removal on morphology and catalytic behaviour, Langmuir 27 (2011) 7909-7916.

[28] F.J. Vidal-Iglesias, J. Solla-Gullón, E. Herrero, V. Montiel, A. Aldaz, J.M. Feliu, Evaluating the ozone cleaning treatment in shape-controlled Pt nanoparticles: Evidences of atomic surface disordering, Electrochem. Commun. 13 (2011) 502-505.

-• This paper demonstrates why particle shape and surface structure must be independently evaluated.

[29] P. Strasser, Catalysts by Platonic design, Science 349 (2015) 379-380.

- This paper summarizes the huge advances on the use of octahedral PtNi nanoparticles towards the oxygen reduction reaction (ORR).

[30] Y.J. Wang, N. Zhao, B. Fang, H. Li, X.T. Bi, H. Wang, Carbon-Supported Pt-Based Alloy Electrocatalysts for the Oxygen Reduction Reaction in Polymer Electrolyte Membrane Fuel Cells: Particle Size, Shape, and Composition Manipulation and Their Impact to Activity, Chem. Rev. 115 (2015) 3433-3467.

[31] D.F. Van Der Vliet, C. Wang, D. Li, A.P. Paulikas, J. Greeley, R.B. Rankin, D. Strmcnik, D. Tripkovic, N.M. Markovic, V.R. Stamenkovic, Unique electrochemical adsorption properties of Pt-skin surfaces, Angew. Chem. Int. Ed. 51 (2012) 3139-3142.

[32] M. Shao, J.H. Odell, S.I. Choi, Y. Xia, Electrochemical surface area measurements of platinum- and palladium-based nanoparticles, Electrochem. Commun. 31 (2013) 4648. 
[33] S. Rudi, C. Cui, L. Gan, P. Strasser, Comparative Study of the Electrocatalytically Active Surface Areas (ECSAs) of Pt Alloy Nanoparticles Evaluated by Hupd and COstripping voltammetry, Electrocatalysis 5 (2014) 408-418.

[34] F.J. Vidal-Iglesias, R.M. Aran-Ais, J. Solla-Gullón, E. Herrero, J.M. Feliu, Electrochemical Characterization of Shape-Controlled Pt Nanoparticles in Different Supporting Electrolytes, ACS Catal. 2 (2012) 901-910.

-• This paper collects cyclic voltammograms obtained with clean shape-controlled Pt nanoparticles in the most conventional supporting electrolytes $\left(\mathrm{H}_{2} \mathrm{SO}_{4}, \mathrm{HClO}_{4}\right.$ or $\mathrm{NaOH})$.

[35] H. Erikson, A. Sarapuu, N. Alexeyeva, K. Tammeveski, J. Solla-Gullón, J.M. FeliU, Electrochemical reduction of oxygen on palladium nanocubes in acid and alkaline solutions, Electrochim. Acta 59 (2012) 329-335.

[36] F.J. Vidal-Iglesias, R.M. Aran-Ais, J. Solla-Gullon, E. Garnier, E. Herrero, A. Aldaz, J.M. Feliu, Shape-dependent electrocatalysis: formic acid electrooxidation on cubic $\mathrm{Pd}$ nanoparticles, Phys. Chem. Chem. Phys. 14 (2012) 10258-10265.

[37] C. Coutanceau, P. Urchaga, S. Brimaud, S. Baranton, Colloidal Syntheses of Shape- and Size-Controlled Pt Nanoparticles for Electrocatalysis, Electrocatalysis 3 (2) (2012) 75-87.

[38] P. Urchaga, S. Baranton, T.W. Napporn, C. Coutanceau, Selective Syntheses and Electrochemical Characterization of Platinum Nanocubes and Nanotetrahedrons/Octahedrons, Electrocatalysis 1 (2010) 3-6.

[39] A. Levendorf, S.G. Sun, Y. Tong, In Situ FT-IR Investigation of Methanol and CO Electrooxidation on Cubic and Octahedral/Tetrahedral Pt Nanoparticles Having Residual PVP, Electrocatalysis 5 (2014) 248-255.

[40] K. M. Koczkur, S. Mourdikoudis, L. Polavarapu, S. E. Skrabalak, Polyvinylpyrrolidone (PVP) in nanoparticle synthesis, Dalton Trans., 44 (2015) 1788317905.

[41] J. Monzó, M.T.M. Koper, P. Rodriguez, Removing polyvinylpyrrolidone from catalytic Pt nanoparticles without modification of superficial order, ChemPhysChem 13 (2012) 709-715.

-• This paper reports the first efficient cleaning protocol for shape-controlled Pt nanoparticles prepared with PVP. 
[42] A.M. Levendorf, D. J. Chen, C.L. Rom, Y. Liu, Y.J. Tong, Electrochemical and in situ ATR-SEIRAS investigations of methanol and CO electro-oxidation on PVP-free cubic and octahedral/tetrahedral Pt nanoparticles, RSC Advances 4 (2014) 2128421293.

[43] H. Yang, Y. Tang, S. Zou, Electrochemical removal of surfactants from Pt nanocubes, Electrochem. Commun. 38 (2014) 134-137.

[44] A. Zalineeva, S. Baranton, C. Coutanceau, Bi-modified palladium nanocubes for glycerol electrooxidation, Electrochem. Commun. 34 (2013) 335-338.

[45] A. Zalineeva, S. Baranton, C. Coutanceau, G. Jerkiewicz, Electrochemical Behavior of Unsupported Shaped Palladium Nanoparticles, Langmuir 31 (2015) 16051609.

[46] C. Susut, Y.J. Tong, Size-Dependent Methanol Electro-oxidation Activity of Pt Nanoparticles with Different Shapes, Electrocatalysis 2 (2011) 75-81.

[47] N. Naresh, F.G.S. Wasim, B.P. Ladewig, M. Neergat, Removal of surfactant and capping agent from $\mathrm{Pd}$ nanocubes (Pd-NCs) using tert-butylamine: Its effect on electrochemical characteristics, J. Mater. Chem. A, 1 (2013) 8553-8559.

[48] N. Nalajala, W.F. Gooty Saleha, B.P. Ladewig, M. Neergat, Sodium borohydride treatment: A simple and effective process for the removal of stabilizer and capping agents from shape-controlled palladium nanoparticles, Chem. Commun. 50 (2014) 9365-9368

[49] M. Luo, Y. Hong, W. Yao, C. Huang, Q. Xu, Q. Wu, Facile removal of polyvinylpyrrolidone (PVP) adsorbates from Pt alloy nanoparticles, J. Mater. Chem. A, 3 (2015) 2770-2775.

[50] R.M. Arán-Ais, F.J. Vidal-Iglesias, J. Solla-Gullon, E. Herrero, J.M. Feliu. Electrochemical characterization of clean shape-controlled Pt nanoparticles prepared in presence of oleylamine/oleic acid. Electroanalysis 27 (2014) 945-956.

-• This paper reports a simple and efficient cleaning protocol for shape-controlled Pt nanoparticles prepared with the oleylamine/oleic acid, one of the most usual system for the preparation of shaped metal alloy nanoparticles.

[51] J. Hernández, J. Solla-Gullón, E. Herrero, J.M. Feliu, A. Aldaz, In Situ Surface Characterization and Oxygen Reduction Reaction on Shape-Controlled Gold Nanoparticles, J Nanosci. Nanotechnol. 9 (2009) 2256-2273. 
[52] Q. Jiang, Z. Jiang, L. Zhang, H. Lin, N. Yang, H. Li, D. Liu, Z. Xie, Z. Tian, Synthesis and high electrocatalytic performance of hexagram shaped gold particles having an open surface structure with kinks, Nano Research 4 (2011) 612-622.

[53] H. Erikson, A. Sarapuu, K. Tammeveski, J. Solla-Gullón, J.M. Feliu, ShapeDependent Electrocatalysis: Oxygen Reduction on Carbon-Supported Gold Nanoparticles, ChemElectroChem 1 (2014) 1338-1347.

[54] J. Monzó, Y. Malewski, F.J. Vidal-Iglesias, J. Solla-Gullón, P. Rodriguez, Electrochemical oxidation of small organic molecules on $\mathrm{Au}$ nanoparticles with preferential surface orientation, ChemElectroChem 2 (2015) 958-962. 